\title{
The obstacle problem for conformal metrics on compact Riemannian manifolds
}

Sijia Bao ${ }^{1 *}$ and Yuming Xing ${ }^{1}$

"Correspondence: Baosj11@163.com

'Department of Mathematics, Harbin Institute of Technology, Harbin, China

\begin{abstract}
We prove a priori estimates up to their second order derivatives for solutions to the obstacle problem of curvature equations on Riemannian manifolds $\left(M^{n}, g\right)$ arising from conformal deformation. With the a priori estimates the existence of a $C^{1,1}$ solution to the obstacle problem with Dirichlet boundary value is obtained by approximation.
\end{abstract}

Keywords: Obstacle problem; A priori estimates; Hessian equations; Viscosity solutions; Riemannian manifolds

\section{Introduction}

Let $\left(M^{n}, g\right)$ be a compact Riemannian manifold of dimension $n \geq 3$ with smooth boundary $\partial M, \bar{M}:=M \cup \partial M$. In conformal geometry, it is interesting to find a complete metric $\tilde{g} \in$ $[g]$, the conformal class of $g$, with which the manifold has prescribed curvature. In general, such conformal deformation can be interpreted by certain partial differential equations. See $[8,13,22,25,26]$ for more details.

In [8], Guan studied the existence of a complete conformal metric $\tilde{g}$ of negative Ricci curvature on $M$ satisfying

$$
f\left(-\lambda\left(\tilde{g}^{-1} \operatorname{Ric}_{\tilde{g}}\right)\right)=\psi \quad \text { in } M
$$

where $\operatorname{Ric}_{\tilde{g}}$ is the Ricci tensor of $\tilde{g}$, and $\lambda\left(\tilde{g}^{-1} \operatorname{Ric}_{\tilde{g}}\right)=\left(\lambda_{1}, \ldots, \lambda_{n}\right)$ are the eigenvalues of $\tilde{g}^{-1} \operatorname{Ric}_{\tilde{g}}$. The transformation formula for the Ricci tensor under conformal deformation $\tilde{g}=e^{2 u} g$ is given by

$$
\frac{1}{n-2} \operatorname{Ric}_{\tilde{g}}=\frac{1}{n-2} \operatorname{Ric}_{g}-\nabla^{2} u-\left(\frac{\Delta u}{n-2}+|\nabla u|^{2}\right) g+d u \otimes d u,
$$

where $\nabla u, \nabla^{2} u$, and $\Delta u$ denote the gradient, Hessian, and Laplacian of $u$ with respect to the metric $g$, respectively. When $f$ is homogenous of degree one, it is easy to verify that equation (1.1) is equivalent to the following form:

$$
f\left(\lambda\left(g^{-1}\left[\nabla^{2} u+\frac{\Delta u}{n-2} g+|\nabla u|^{2} g-d u \otimes d u-\frac{\operatorname{Ric}_{g}}{n-2}\right]\right)\right)=\frac{\psi(x)}{n-2} e^{2 u} .
$$


In this paper, we study the obstacle problem of equation (1.2). More generally, let

$$
T[u]:=\nabla^{2} u+s d u \otimes d u+\left(\gamma \Delta u-\frac{t}{2}|\nabla u|^{2}\right) g+\chi
$$

where $\chi$ is a smooth $(0,2)$ tensor, $\gamma>0$ is a constant, and $s, t \in \mathbb{R}$. We consider the following equation:

$$
\max \left\{u-h,-\left(f\left(\lambda\left(g^{-1} T[u]\right)\right)-\psi[u]\right)\right\}=0 \quad \text { in } M
$$

with the Dirichlet boundary condition

$$
u=\varphi \quad \text { on } \partial M
$$

where $h \in C^{3}(\bar{M}), \varphi \in C^{4}(\partial M), h>\varphi$ on $\partial M, \psi[u]=\psi(x, u)$ is a positive function in $C^{3}(\bar{M} \times \mathbb{R})$.

Equations as (1.1) and (1.3) are the Hessian equations, which were well studied by many authors such as $[2,7,9-12,23,24]$. Generally, $f \in C^{2}(\Gamma) \cap C^{0}(\bar{\Gamma})$ is a symmetric function of $\lambda \in \mathbb{R}^{n}$, defined in an open, convex, and symmetric cone $\Gamma \varsubsetneqq \mathbb{R}^{n}$, with vertex at the origin, which contains the positive cone: $\Gamma_{n}^{+}:=\left\{\lambda \in \mathbb{R}^{n}\right.$ : each component $\left.\lambda_{i}>0\right\}$ and satisfies the following fundamental structure conditions:

$$
f_{i} \equiv \frac{\partial f}{\partial \lambda_{i}}>0 \quad \text { in } \Gamma, 1 \leq i \leq n,
$$

$f$ is a concave function,

and

$$
f>0 \text { in } \Gamma, \quad f=0 \text { on } \partial \Gamma .
$$

Here, for convenience, we also assume that

$$
f \text { is homogeneous of degree one. }
$$

We observe that by the concavity and homogeneity of $f$,

$$
\sum f_{i}(\lambda)=f(\lambda)+\sum f_{i}(\lambda)\left(1-\lambda_{i}\right) \geq f(1, \ldots, 1)>0 \quad \text { in } \Gamma .
$$

Important classes of $f$ are the elementary symmetric functions and their quotients, i.e.,

$$
f(\lambda)=\left(\sigma_{k}\right)^{\frac{1}{k}}(\lambda):=\left(\sum_{1 \leq i_{1}<\cdots<i_{k} \leq n} \lambda_{i_{1}} \cdots \lambda_{i_{k}}\right)^{\frac{1}{k}}, \quad 1 \leq k \leq n,
$$

and

$$
f(\lambda)=\left(\frac{\sigma_{k}}{\sigma_{l}}\right)^{\frac{1}{k-l}}, \quad 0 \leq l<k \leq n
$$


Let $F$ be defined by $F(r)=f(\lambda(r))$ for $r=\left\{r_{i j}\right\} \in \mathcal{S}^{n \times n}$ with $\lambda(r) \in \Gamma$, where $\mathcal{S}^{n \times n}$ is the set of $n \times n$ symmetric matrices. It is shown in [2] that (1.5) implies $F$ is an elliptic operator and (1.6) ensures that $F$ is concave.

A function $u \in C^{2}(M)$ is called admissible at $x \in M$ if $\lambda\left(g^{-1} T[u]\right)(x) \in \Gamma$, and we call it admissible in $M$ when it is admissible at each $x$ in $M$. In this paper, we prove the existence of an admissible viscosity solution of $(1.3)$ and $(1.4)$ in $C^{1,1}(\bar{M})$ (see $[1,3]$ for the definition of viscosity solutions).

Many authors have studied various obstacle problems. In [6], Gerhardt considered a hypersurface bounded from below by an obstacle with prescribed mean curvature in $\mathbb{R}^{n}$. Lee [17] considered the obstacle problem for the Monge-Ampère equation (i.e., $f=\left(\sigma_{n}\right)^{\frac{1}{n}}$ ) for the case that $T[u]=D^{2} u, \psi \equiv 1$, and $\varphi \equiv 0$, and proved the $C^{1,1}$ regularity of the viscosity solution in a strictly convex domain in $\mathbb{R}^{n}$. Xiong and Bao [27] extended the work of Lee to a nonconvex domain in $\mathbb{R}^{n}$ with general $\psi$ and $\varphi$ under additional assumptions. Bao, Dong, and Jiao treated a class of obstacle problems in [1] assuming that $T[u]=\nabla^{2} u+A(x, u, \nabla u)$, under a certain technical assumption. Because of the term $\gamma \Delta u(\gamma>0)$, here we only need a minimal amount of assumptions. For other works, see $[4,14,15,18-21]$.

Our main result is the following theorem.

Theorem 1.1 Assume that (1.5)-(1.8) and either the following condition

$$
\lim _{z \rightarrow+\infty} \psi(x, z)=+\infty, \quad \forall x \in \bar{M}
$$

or

$$
\frac{2 s-n t}{1+n \gamma}<2 \lambda_{1}
$$

hold, where $\lambda_{1}$ is the first eigenvalue of the problem

$$
\begin{cases}\Delta u+\lambda(\operatorname{tr} \chi)^{+} u=0 & \text { on } \bar{M}, \\ u=0 & \text { on } \partial M\end{cases}
$$

$\left(\lambda_{1}=+\infty\right.$ if $\left.\operatorname{tr} \chi \leq 0\right)$. Then there exists a viscosity solution $u \in C^{1,1}(\bar{M})$ to (1.3) and (1.4), if there exists a subsolution $\underline{u} \in C^{0}(\bar{M}) \cap C^{1}\left(\bar{M}_{\delta}\right)$ for some $\delta>0$ such that

$$
\begin{cases}f\left(\lambda\left(g^{-1} T[\underline{u}]\right)\right) \geq \psi[\underline{u}], & \text { in } M, \\ \underline{u}=\varphi, & \text { on } \partial M, \\ \underline{u} \leq h, & \text { in } M,\end{cases}
$$

where $M_{\delta}=\{x \in M: \operatorname{dist}(x, \partial M) \leq \delta\}$. Moreover, we have that $u \in C^{3, \alpha}(E)$ for any $\alpha \in(0,1)$, and $f\left(\lambda\left(g^{-1} T[u]\right)\right)=\psi[u]$ in $E$, where $E:=\{x \in M: u(x)<h(x)\}$.

Remark 1.2 (1.10), as well as (1.11), is used in Lemma 3.2 to derive an upper bound for $u$. Assumption (1.13) is just applied to derive a lower bound for $u$ on $M$ and $\nabla_{v} u$ on $\partial M$, where $v$ is the interior unit normal to $\partial M$. 
Remark 1.3 We can construct some subsolutions of (1.2) satisfying (1.13) as in [15] following ideas from [2] and [7] since

$$
|\nabla u|^{2} g-d u \otimes d u
$$

is positive definite and that we can obtain a priori upper bound of any admissible function (Lemma 3.2) under additional conditions that there exists a sufficiently large number $R>0$ such that at each point $x \in \partial M$,

$$
\left(\kappa_{1}, \ldots, \kappa_{n-1}, R\right) \in \Gamma
$$

where $\kappa_{1}, \ldots, \kappa_{n-1}$ are the principal curvatures of $\partial M$ with respect to the interior normal, and that for every $C>0$ and every compact set $K$ in $\Gamma$ there is a number $R=R(C, K)$ such that

$$
f(R \lambda) \geq C \quad \text { for all } \lambda \in K
$$

We use a penalization technique to prove the existence of viscosity solutions to (1.3) and (1.4). We shall consider the following singular perturbation problem:

$$
\begin{cases}f\left(\lambda\left(g^{-1} T[u]\right)\right)=\psi[u]+\beta_{\varepsilon}(u-h) & \text { in } M, \\ u=\varphi & \text { on } \partial M\end{cases}
$$

where the penalty function $\beta_{\varepsilon} \in C^{2}(\mathbb{R})$ satisfies

$$
\begin{aligned}
& \beta_{\varepsilon}, \beta_{\varepsilon}^{\prime}, \beta_{\varepsilon}^{\prime \prime} \geq 0 \quad \text { on } \mathbb{R}, \beta_{\varepsilon}(z)=0, \text { whenever } z \leq 0 ; \\
& \beta_{\varepsilon}(z) \rightarrow \infty \quad \text { as } \varepsilon \rightarrow 0^{+}, \text {whenever } z>0 .
\end{aligned}
$$

An example given in [27] is

$$
\beta_{\varepsilon}(z)= \begin{cases}0, & z \leq 0 \\ z^{3} / \varepsilon, & z>0\end{cases}
$$

for $\varepsilon \in(0,1)$. Observe that $\underline{u}$ is also a subsolution to (1.16).

Let

$$
\mathcal{U}=\left\{u_{\varepsilon} \mid u_{\varepsilon} \in C^{4}(\bar{M}) \text { is an admissible solution of (1.16) with } u_{\varepsilon} \geq \underline{u} \text { on } \bar{M}\right\} \text {. }
$$

We aim to derive the uniform bound

$$
\left|u_{\varepsilon}\right|_{C^{2}(\bar{M})} \leq C
$$

for $u_{\varepsilon} \in U$, where $C$ is independent of $\varepsilon$. After establishing (1.19), the equation (1.16) becomes uniformly elliptic by (1.7). By Evans-Krylov [5], [16] theorem, we can derive the $C^{2, \alpha}$ estimates (which may depend on $\varepsilon$ ) of $u_{\varepsilon}$. Higher estimates can be derived by 
Schauder theory. Following the proof as in [8] or [1], we can prove there exists an admissible solution $u_{\varepsilon}$ to (1.16). Then we can conclude by (1.19) that there exists a viscosity solution $u \in C^{1,1}(\bar{M})$ to $(1.3)$ and $(1.4)$, see $[1,27]$.

Thus, our main work is focused on the a priori estimates for admissible solutions up to their second order derivatives. In Sect. 2, we achieve the estimates for second order derivatives. Finally, we end this paper with gradient and $C^{0}$ estimates in Sect. 3.

\section{Estimates for second order derivatives}

In this section, we prove a priori estimates of second order derivatives for admissible solutions. From now on, we drop the subscript $\varepsilon$ when there is no possible confusion.

Theorem 2.1 Assume that $f$ satisfies (1.5)-(1.8) and $u \in C^{4}(\bar{M})$ is an admissible solution to (1.16). Then

$$
\sup _{M}\left|\nabla^{2} u\right| \leq C\left(1+\sup _{\partial M}\left|\nabla^{2} u\right|\right)
$$

where $C$ depends on $|u|_{C^{1}(\bar{M})}$ and other known data.

Proof Set

$$
W(x)=\max _{\xi \in T_{x} M,|\xi|=1}\left(\nabla_{\xi \xi} u+s\left|\nabla_{\xi} u\right|^{2}\right) e^{\phi}, \quad x \in \bar{M}
$$

where $\phi$ is a function to be determined. Assume that $W$ is achieved at an interior point $x_{0} \in M$ and a unit direction $\xi \in T_{x_{0}} M$. Choose a smooth orthonormal local frame $e_{1}, \ldots, e_{n}$ about $x_{0}$ such that $\xi=e_{1}, \nabla_{i} e_{j}\left(x_{0}\right)=0$ and that $T_{i j}\left(x_{0}\right)$ is diagonal. We write $G=\nabla_{11} u+$ $s\left|\nabla_{1} u\right|^{2}$. Assume $G\left(x_{0}\right)>0$ (otherwise we are done).

At the point $x_{0}$, where the function $\log G+\phi$ (defined near $x_{0}$ ) attains its maximum, we have

$$
\frac{\nabla_{i} G}{G}+\nabla_{i} \phi=0, \quad i=1, \ldots, n,
$$

and

$$
\frac{\nabla_{i i} G}{G}-\left(\frac{\nabla_{i} G}{G}\right)^{2}+\nabla_{i i} \phi \leq 0
$$

By (2.3) we have

$$
F^{i i}\left(\nabla_{i i} G+G \nabla_{i i} \phi-G\left|\nabla_{i} \phi\right|^{2}\right) \leq 0
$$

and

$$
\Delta G+G \Delta \phi-G|\nabla \phi|^{2} \leq 0
$$

Since $\gamma>0$, we obtain

$$
F^{i i}\left(\nabla_{i i} G+\gamma \Delta G+G \nabla_{i i} \phi+\gamma G \Delta \phi-G\left|\nabla_{i} \phi\right|^{2}-\gamma G|\nabla \phi|^{2}\right) \leq 0 .
$$


By calculation, we get

$$
\nabla_{i} G=\nabla_{i 11} u+2 s \nabla_{1} u \nabla_{i 1} u
$$

and

$$
\nabla_{i i} G=\nabla_{i i 11} u+2 s\left(\left|\nabla_{i 1} u\right|^{2}+\nabla_{1} u \nabla_{i i 1} u\right)
$$

Recall the formula for interchanging order of covariant derivatives

$$
\nabla_{i j k} v-\nabla_{k i j} v=R_{k i j}^{l} \nabla_{l} v
$$

and

$$
\begin{aligned}
\nabla_{i j k l} v-\nabla_{k l i j} v= & R_{l j k}^{m} \nabla_{i m} v+\nabla_{i} R_{l j k}^{m} \nabla_{m} v+R_{l i k}^{m} \nabla_{j m} v \\
& +R_{j i k}^{m} \nabla_{l m} v+R_{j i l}^{m} \nabla_{k m} v+\nabla_{k} R_{j i l}^{m} \nabla_{m} v .
\end{aligned}
$$

It follows from (2.10)

$$
\nabla_{i i} G \geq \nabla_{11 i i} u+2 s\left(\left|\nabla_{i 1} u\right|^{2}+\nabla_{1} u \nabla_{1 i i} u\right)-C(1+G)
$$

and

$$
\begin{aligned}
\nabla_{i i} G+\gamma \Delta G \geq & \nabla_{11 i i} u+2 s\left(\left|\nabla_{i 1} u\right|^{2}+\nabla_{1} u \nabla_{1 i i} u\right)+\gamma \nabla_{11}(\Delta u) \\
& +2 s \gamma\left(\left|\nabla_{i 1} u\right|^{2}+\nabla_{1} u \nabla_{1}(\Delta u)\right)-C(1+G) .
\end{aligned}
$$

Differentiating equation (1.16) once at $x_{0}$, we obtain for $1 \leq k \leq n$,

$$
\nabla_{k} F=F^{i i} \nabla_{k} T_{i i}=\psi_{x_{k}}+\psi_{z} \nabla_{k} u+\nabla_{k} \beta_{\varepsilon}(u-h) .
$$

It is easy to see that

$$
\begin{aligned}
F^{i i} \nabla_{1}\left(\nabla_{i i} u+\gamma \Delta u\right) & =F^{i i} \nabla_{1}\left(T_{i i}[u]-s\left|\nabla_{i} u\right|^{2}+\frac{t}{2}|\nabla u|^{2}-\chi_{i i}\right) \\
& \geq \nabla_{1} F-2 s F^{i i} \nabla_{i} u \nabla_{1 i} u+t \nabla_{k} u \nabla_{1 k} u \sum_{i} F^{i i}-\sum_{i} F^{i i}
\end{aligned}
$$

and that

$$
\begin{aligned}
F^{i i} \nabla_{11}\left(\nabla_{i i} u+\gamma \Delta u\right)= & F^{i i} \nabla_{11}\left(T_{i i}[u]-s\left|\nabla_{i} u\right|^{2}+\frac{t}{2}|\nabla u|^{2}-\chi_{i i}\right) \\
\geq & F^{i i} \nabla_{11} T_{i i}[u]-2 s F^{i i}\left(\nabla_{i} u \nabla_{11 i} u+\left|\nabla_{1 i} u\right|^{2}\right) \\
& +t \sum_{k}\left(\nabla_{k} u \nabla_{11 k} u+\left|\nabla_{1 k} u\right|^{2}\right) \sum_{i} F^{i i}-C \sum_{i} F^{i i}
\end{aligned}
$$


With (2.9) we see

$$
\begin{aligned}
2 s \nabla_{i} u \nabla_{11 i} u & \leq 2 s \nabla_{i} u\left(\nabla_{i} G-2 s \nabla_{1} u \nabla_{i 1} u\right)+C \\
& \leq-4 s^{2} \nabla_{i} u \nabla_{1} u \nabla_{i 1} u+C(1+G|\nabla \phi|),
\end{aligned}
$$

and similarly

$$
t \nabla_{k} u \nabla_{11 k} u \geq-2 s t \nabla_{k} u \nabla_{1} u \nabla_{k 1} u-C(1+G|\nabla \phi|) .
$$

With (2.12), (2.14)-(2.17), and the concavity of $F$, we derive

$$
\begin{aligned}
F^{i i}\left(\nabla_{i i} G+\gamma \Delta G\right) \geq & \nabla_{11} F+2 s \nabla_{1} u \nabla_{1} F+(2 s \gamma+t) \sum_{k}\left|\nabla_{1 k} u\right|^{2} \sum F^{i i} \\
& -C\left(G+G|\nabla \phi|+\sum_{j, k}\left|\nabla_{j k} u\right|\right) \\
\geq & \nabla_{11} F+2 s \nabla_{1} u \nabla_{1} F-C\left(G^{2}+G|\nabla \phi|\right) .
\end{aligned}
$$

By (1.9) and $\beta_{\varepsilon}^{\prime \prime}>0$ it follows from (2.6) and (2.18) that

$$
\begin{aligned}
& F^{i i}\left(\nabla_{i i} \phi-\left|\nabla_{i} \phi\right|^{2}\right)+\gamma\left(\Delta \phi-|\nabla \phi|^{2}\right) \sum F^{i i} \\
& \quad \leq C(G+|\nabla \phi|) \sum F^{i i}+\left(\frac{C}{G}-1\right) \beta_{\varepsilon}^{\prime}(u-h) .
\end{aligned}
$$

Let

$$
\phi:=\eta(w)=\left(1-\frac{w}{2 a}\right)^{-1 / 2}, \quad w=\frac{|\nabla u|^{2}}{2}
$$

where $a>\sup _{M} w$ is a constant to be determined. We have

$$
1 \leq \eta<\sqrt{2}, \quad \eta^{\prime}=\frac{\eta^{3}}{4 a}, \quad \eta^{\prime \prime}=\frac{3 \eta^{\prime 2}}{\eta}
$$

and

$$
\nabla_{i i} \phi-\left|\nabla_{i} \phi\right|^{2}=\eta^{\prime} \nabla_{i i} w+\left(\eta^{\prime \prime}-\eta^{\prime 2}\right)\left|\nabla_{i} w\right|^{2} \geq \eta^{\prime} \nabla_{i i} w
$$

Next, by (2.14)

$$
\begin{aligned}
& F^{i i}\left(\nabla_{i i} w+\gamma \Delta w\right) \\
& \quad=F^{i i}\left(\sum_{l}\left|\nabla_{i l} u\right|^{2}+\gamma \sum_{k, l}\left|\nabla_{k l} u\right|^{2}\right)+F^{i i} \nabla_{l} u\left(\nabla_{i i l} u+\gamma \Delta\left(\nabla_{l} u\right)\right) \\
& \quad \geq F^{i i} \nabla_{l} u\left(\nabla_{l i i} u+\gamma \sum_{k} \nabla_{l k k} u\right)+\left(\gamma G^{2}-C G\right) \sum F^{i i} \\
& \quad \geq-C \beta_{\varepsilon}^{\prime}(u-h)+\left(\gamma G^{2}-C G\right) \sum F^{i i} .
\end{aligned}
$$


Combining (2.19), (2.20), (2.21), and $|\nabla \phi| \leq C \eta^{\prime} G$, we have

$$
\eta^{\prime}\left(\gamma G^{2}-C G\right) \sum F^{i i} \leq C\left(G+\eta^{\prime} G\right) \sum F^{i i}+\left(\frac{C}{G}-1+C \eta^{\prime}\right) \beta_{\varepsilon}^{\prime}(u-h) .
$$

We could assume that $G \geq 2 C$. When $a>2 C$, the coefficient of $\beta_{\varepsilon}^{\prime}(u-h)$ is negative. Then we can derive $G \leq \frac{4 a C}{\gamma}$.

To derive the boundary estimates for $\nabla^{2} u$, we note that $\operatorname{tr}\left(s d u \otimes d u-\frac{t}{2}|\nabla u|^{2} g+\chi\right) \leq C$ on $\bar{M}$, where $C$ is independent of $\varepsilon$, though it may depend on $|u|_{C^{1}(\bar{M})}$. As in $[1,4]$, let $H$ be the solution to

$$
\begin{cases}(1+n \gamma) \Delta H+C=0 & \text { in } M, \\ H=\varphi & \text { on } \partial M .\end{cases}
$$

Then we have $u \leq H$ in $M$ by the maximum principle and $\beta_{\varepsilon}(u-h) \equiv 0$ in $M_{\delta}=\{x \in M$ : $\operatorname{dist}(x, \partial M) \leq \delta\}$, where $\delta$ is sufficiently small. Thus,

$$
\begin{cases}f\left(\lambda\left(g^{-1} T[u]\right)\right)=\psi[u] & \text { in } M_{\delta} \\ u=\varphi & \text { on } \partial M\end{cases}
$$

By the same arguments of Sect. 4 in [8], we obtain that

$$
\sup _{\partial M}\left|\nabla^{2} u\right| \leq C
$$

where $C$ depends on $|u|_{C^{1}(\bar{M})}$ and other known data.

Combining (2.1) and (2.24), we therefore get the full estimates for second order derivatives.

\section{Gradient estimates, maximum principle, and existence}

For the gradient estimates, we have the following theorem.

Theorem 3.1 Assume that (1.5)-(1.8) hold. Let $u \in C^{3}(\bar{M})$ be an admissible solution to (1.16). Then

$$
\sup _{M}|\nabla u| \leq C\left(1+\sup _{\partial M}|\nabla u|\right)
$$

where $C$ depends on $|u|_{C^{0}(\bar{M})}$ and other known data.

Proof Suppose that $w e^{\phi}$, where $w=\frac{|\nabla u|^{2}}{2}$ and $\phi=\phi(u)$ to be determined satisfying that $\phi^{\prime}(u)>0$, achieves a maximum at an interior point $x_{0} \in M$. As before, we choose a smooth orthonormal local frame $e_{1}, \ldots, e_{n}$ about $x_{0}$ such that $\nabla_{e_{i}} e_{j}=0$ at $x_{0}$ and $\left\{T_{i j}\left(x_{0}\right)\right\}$ is diagonal. Differentiating $w e^{\phi}$ at $x_{0}$ twice, we have

$$
\nabla_{i} w+w \nabla_{i} \phi=0
$$


and

$$
\nabla_{i i} w-w\left(\nabla_{i} \phi\right)^{2}+w \nabla_{i i} \phi \leq 0
$$

Differentiating $w$, we see

$$
\nabla_{i} w=\sum_{k} \nabla_{k} u \nabla_{i k} u, \quad \nabla_{i i} w=\sum_{k}\left(\nabla_{i k} u\right)^{2}+\sum_{k} \nabla_{k} u \nabla_{i i k} u
$$

Using (3.2) it follows from (3.3) that

$$
F^{i i}\left(\delta_{k l}-\frac{\nabla_{k} u \nabla_{l} u}{2 w}\right) \nabla_{i k} u \nabla_{i l} u+F^{i i} \nabla_{k} u \nabla_{i i k} u-w F^{i i}\left(\frac{\left(\nabla_{i} \phi\right)^{2}}{2}-\nabla_{i i} \phi\right) \leq 0
$$

and

$$
\sum_{i, k, l}\left(\delta_{k l}-\frac{\nabla_{k} u \nabla_{l} u}{2 w}\right) \nabla_{i k} u \nabla_{i l} u+\nabla_{k} u \Delta\left(\nabla_{k} u\right)-\frac{w}{2}|\nabla \phi|^{2}+w \Delta \phi \leq 0 .
$$

Note that the first term in (3.4) and (3.5) is nonnegative. Multiply $\gamma \sum F^{i i}$ to (3.5) and add what we got to (3.4). Thus, by (2.9) we obtain

$$
\begin{gathered}
F^{i i} \nabla_{k} u\left(\nabla_{k i i} u+\gamma \nabla_{k} \Delta u\right)-\frac{w}{2} F^{i i}\left(\left|\nabla_{i} \phi\right|^{2}+\gamma|\nabla \phi|^{2}\right) \\
+w F^{i i}\left(\nabla_{i i} \phi+\gamma \Delta \phi\right) \leq C|\nabla u|^{2} \sum F^{i i} .
\end{gathered}
$$

Now we compute the first term in (3.6). Firstly, we have

$$
\nabla_{i} \phi=\phi^{\prime} \nabla_{i} u, \quad \nabla_{i i} \phi=\phi^{\prime} \nabla_{i i} u+\phi^{\prime \prime}\left(\nabla_{i} u\right)^{2} .
$$

Using (3.2), we easily get that

$$
\begin{aligned}
F^{i i} & \nabla_{k} u\left(\nabla_{k i i} u+\gamma \nabla_{k} \Delta u\right) \\
& =F^{i i} \nabla_{k} u \nabla_{k}\left(T_{i i}-s\left|\nabla_{i} u\right|^{2}+\frac{t}{2}|\nabla u|^{2}-\chi_{i i}\right) \\
& =\nabla_{k} u \nabla_{k}\left(\psi+\beta_{\varepsilon}\right)+w \phi^{\prime} F^{i i}\left(2 s\left|\nabla_{i} u\right|^{2}-t|\nabla u|^{2}\right)-F^{i i} \nabla_{k} u \nabla_{k} \chi_{i i} .
\end{aligned}
$$

By the homogeneity of $F$, we also get

$$
\begin{aligned}
F^{i i} & \left(\nabla_{i i} \phi+\gamma \Delta \phi\right) \\
& =\phi^{\prime \prime} F^{i i}\left(\left|\nabla_{i} u\right|^{2}+\gamma|\nabla u|^{2}\right)+\phi^{\prime} F^{i i}\left(T_{i i}-s\left|\nabla_{i} u\right|^{2}+\frac{t}{2}|\nabla u|^{2}-\chi_{i i}\right) \\
& =\phi^{\prime \prime} F^{i i}\left(\left|\nabla_{i} u\right|^{2}+\gamma|\nabla u|^{2}\right)+\phi^{\prime}\left(F-s F^{i i}\left|\nabla_{i} u\right|^{2}+\frac{t}{2} F^{i i}|\nabla u|^{2}-F^{i i} \chi_{i i}\right) .
\end{aligned}
$$


According to (3.7) and (3.8), it follows from (3.6)

$$
\begin{aligned}
& \gamma|\nabla u|^{2}\left(\phi^{\prime \prime}-\frac{1}{2}\left(\phi^{\prime}\right)^{2}-\frac{t}{2 \gamma} \phi^{\prime}\right) \sum F^{i i}+\left(\phi^{\prime \prime}-\frac{1}{2}\left(\phi^{\prime}\right)^{2}+s \phi^{\prime}\right) F^{i i}\left(\nabla_{i} u\right)^{2} \\
& \leq-\phi^{\prime}\left(\psi+\beta_{\varepsilon}-F^{i i} \chi_{i i}\right)+C \sum F^{i i}-\frac{\nabla_{k} u \nabla_{k}\left(\psi+\beta_{\varepsilon}\right)}{w} \\
& \leq-\left(\phi^{\prime} \beta_{\varepsilon}+\frac{\nabla_{k} u \nabla_{k}(u-h) \beta_{\varepsilon}^{\prime}}{w}\right)+C \sum F^{i i}+C
\end{aligned}
$$

Let

$$
\phi(u)=v^{-a}, \quad v=1-u+\sup _{M} u .
$$

We have

$$
\phi^{\prime}(u)=a v^{-a-1}, \quad \phi^{\prime \prime}(u)=\frac{(a+1) \phi^{\prime}}{v}
$$

and

$$
\phi^{\prime \prime}-\frac{1}{2}\left(\phi^{\prime}\right)^{2}=\phi^{\prime}\left(\frac{a+1}{v}-\frac{a v^{-a}}{2 v}\right) \geq \frac{\phi^{\prime} a}{2 v}>0
$$

since $v^{-a} \leq 1$. When $\left|\nabla u\left(x_{0}\right)\right|$ is sufficiently large, we see $\nabla_{k} u \nabla_{k}(u-h)>0$. Hence we have that the first term on the right-hand side of (3.9) is negative as $\beta_{\varepsilon}, \beta_{\varepsilon}^{\prime}>0$. From (3.9) and (1.9) when $a$ is sufficiently large, we then obtain that

$$
\frac{\phi^{\prime} a \gamma|\nabla u|^{2}}{4 v} \leq C
$$

from which we conclude that (3.1) holds.

In order to prove (1.19), it remains to bound $\sup _{M}|u|+\sup _{\partial M}|\nabla u|$. We quote two lemmas in [8], the ingredients of whose proofs are the maximum principle.

Lemma 3.2 If either (1.10) or (1.11) holds, then any admissible solution u of (1.16) admits the a priori bound

$$
\sup _{M} u \leq c_{0}
$$

Lemma 3.3 If $u$ is admissible such that $\operatorname{tr} T[u] \geq 0$ and $|u|_{C^{0}(M)} \leq \mu$, then

$$
\sup _{\partial M} \nabla_{\nu} u \leq c_{1}(\mu)
$$

where $v$ is the interior unit normal to $\partial M$.

Now with the above two lemmas and the fact $\nabla_{\nu} u \geq \nabla_{\nu} \underline{u}$ on $\partial M$ when $u \in \mathcal{U}$, we then have the following. 
Theorem 3.4 Suppose that (1.5)-(1.8), and either (1.10) or (1.11) hold. Then, for $u \in \mathcal{U}$, (1.19) holds.

Therefore, the uniform estimates (1.19) ensure that there exist a subsequence $\left\{u_{\varepsilon_{k}}\right\}$ of $\left\{u_{\varepsilon}\right\}$ and a function $u \in C^{1,1}(\bar{M})$ such that $u_{\varepsilon_{k}} \rightarrow u$ in $M$ as $\varepsilon_{k} \rightarrow 0$. It is easy to verify that $u$ satisfies (1.3) and (1.4) and $u \in C^{3, \alpha}(E)$ for any $\alpha \in(0,1)$. Consequently, Theorem 1.1 is established.

\section{Funding}

The research was supported by the National Natural Science Foundation of China (No. 11771107).

\section{Competing interests}

The authors declare that they have no competing interests.

\section{Authors' contributions}

All authors contributed equally to the writing of this paper. All authors read and approved the final manuscript.

\section{Publisher's Note}

Springer Nature remains neutral with regard to jurisdictional claims in published maps and institutional affiliations.

Received: 16 April 2018 Accepted: 25 August 2018 Published online: 12 September 2018

\section{References}

1. Bao, G.-J., Dong, W.-S., Jiao, H.-M.: Regularity for an obstacle problem of Hessian equations on Riemannian manifolds. J. Differ. Equ. 258, 696-716 (2015)

2. Caffarelli, L.A., Nirenberg, L., Spruck, J.: The Dirichlet problem for nonlinear second order elliptic equations III, functions of the eigenvalues of the Hessian. Acta Math. 155, 261-301 (1985)

3. Crandall, M., Ishii, H., Lions, P.: User's guide to viscosity solutions of second order partial differential equations. Bull. Am. Math. Soc. 27, 1-67 (1992)

4. Dong, W.-S., Wang, T.-T., Bao, G.-J.: A priori estimates for the obstacle problem of Hessian type equations on Riemannian manifolds. Commun. Pure Appl. Anal. 15, 1769-1780 (2016)

5. Evans, L.C.: Classical solutions of fully nonlinear, convex, second order elliptic equations. Commun. Pure Appl. Math. 35, 333-363 (1982)

6. Gerhardt, C.: Hypersurfaces of prescribed mean curvature over obstacles. Math. Z. 133, 169-185 (1973)

7. Guan, B.: The Dirichlet problem for Hessian equations on Riemannian manifolds. Calc. Var. Partial Differ. Equ. 8, 45-69 (1999)

8. Guan, B.: Complete conformal metrics of negative Ricci curvature on compact manifolds with boundary. Int. Math. Res. Not. 2008, rnn105 (2008) Addendum, IMRN 2009 (2009), 4354-4355, rnp166.

9. Guan, B.: Second order estimates and regularity for fully nonlinear elliptic equations on Riemannian manifolds. Duke Math. J. 163, 1491-1524 (2014)

10. Guan, B.: The Dirichlet problem for fully nonlinear elliptic equations on Riemannian manifolds. arXiv:1403.2133

11. Guan, B., Jiao, H.-M.: Second order estimates for Hessian type fully nonlinear elliptic equations on Riemannian manifolds. Calc. Var. Partial Differ. Equ. 54, 2693-2712 (2015)

12. Guan, B., Jiao, H.-M.: The Dirichlet problem for Hessian type fully nonlinear elliptic equations on Riemannian manifolds. Discrete Contin. Dyn. Syst. 36, 701-714 (2016)

13. Guan, P.-F., Wang, G.-F.: Local estimates for a class of fully nonlinear equations arising from conformal geometry. Int. Math. Res. Not. 26, 1413-1532 (2003)

14. Jiao, H.-M.: $C^{1,1}$ regularity for an obstacle problem of Hessian equations on Riemannian manifolds. Proc. Am. Math. Soc. 144, 3441-3453 (2016)

15. Jiao, H.-M., Wang, Y.: The obstacle problem for Hessian equations on Riemannian manifolds. Nonlinear Anal. TMA 95, 543-552 (2014)

16. Krylov, N.V.: Boundedly inhomogeneous elliptic and parabolic equations in a domain. Izvestia Math. Ser. 47, 75-108 (1983)

17. Lee, K.: The obstacle problem for Monge-Ampère equation. Commun. Partial Differ. Equ. 26, 33-42 (2001)

18. Liu, J.-K., Zhou, B.: An obstacle problem for a class of Monge-Ampère type functionals. J. Differ. Equ. 254, 1306-1325 (2013)

19. Oberman, A.: The convex envelope is the solution of a nonlinear obstacle problem. Proc. Am. Math. Soc. 135, 1689-1694 (2007)

20. Oberman, A., Silvestre, L.: The Dirichlet problem for the convex envelope. Trans. Am. Math. Soc. 363, 5871-5886 (2011)

21. Savin, O.: The obstacle problem for Monge-Ampere equation. Calc. Var. Partial Differ. Equ. 22, 303-320 (2005)

22. Schoen, R.: Conformal deformation of a Riemannian metric to constant scalar curvature. J. Differ. Geom. 20, 479-495 (1984)

23. Trudinger, N.S.: On the Dirichlet problem for Hessian equations. Acta Math. 175, 151-164 (1995)

24. Urbas, J.: Hessian equations on compact Riemannian manifolds. In: Nonlinear Problems in Mathematical Physics and Related Topics, II, pp. 367-377. Kluwer/Plenum, New York (2002) 
25. Viaclovsky, J.A.: Conformal geometry, contact geometry, and the calculus of variations. Duke Math. J. 101, 283-316 (2000)

26. Viaclovsky, J.A.: Conformal Geometry and Fully Nonlinear Equations. Nankai Tracts in Mathematics, vol. 11, pp. 435-460. World Scientific, Hackensack (2006)

27. Xiong, J.-G., Bao, J.-G.: The obstacle problem for Monge-Ampère type equations in non-convex domains. Commun. Pure Appl. Anal. 10, 59-68 (2011)

Submit your manuscript to a SpringerOpen ${ }^{\circ}$ journal and benefit from:

- Convenient online submission

- Rigorous peer review

- Open access: articles freely available online

- High visibility within the field

- Retaining the copyright to your article

Submit your next manuscript at $\boldsymbol{~ s p r i n g e r o p e n . c o m ~}$ 